\title{
A Young Lady with Acute Pulmonary Embolism after Caesarean Section - A Case Report
}

\author{
TAIMUR SDM ${ }^{\mathrm{a}}$, HAQ MM ${ }^{\mathrm{a}}, \mathrm{KHAN} \mathrm{SR}^{\mathrm{a}}, \mathrm{KABIR} \mathrm{CMS}^{\mathrm{a}}$, RAHMAN H${ }^{\mathrm{a}}, \mathrm{KARIM} \mathrm{MR}^{\mathrm{a}}$, GOMES HI $^{\mathrm{a}}$, \\ SALAHUDDIN $\mathrm{M}^{\mathrm{a}}$
}

\begin{abstract}
:
The presentation of acute dyspnea after pregnancy is rare, but should bring a number of important conditions to the mind of the attending physician. Pulmonary embolism, amniotic fluid embolism, pneumonia,aspiration pneumonia and pulmonary edema are some of the potentially devastating causes that must be considered. We report a 29 years young lady who presented with progressive
\end{abstract}

\section{Introduction:}

Pregnancy-related venous thromboembolism (VTE) is a major cause of maternal morbidity and mortality in Western countries. ${ }^{1,2}$ Normal pregnancy is associated with a manifest shift of the coagulation and fibrinolytic systems towards hypercoagulability. Although these changes are of physiological importance in minimizing the risk of blood loss during delivery, they also increase the risk of thromboembolism.

Some major predisposing factors are increasing maternal age, operative delivery, immobilization, obesity, heart disease, malignancy, Caucasian descent, a history of thrombosis, thrombophilia, and familial thrombosis. The incidence of VTE is reported to be about 13/10,000 pregnancies, about half occurring before delivery and the other half in the puerperal period. ${ }^{3,4}$ Thus, during a six-week span, the risk of VTE increases roughly 5-fold as compared to the antepartum period. In addition, it has been shown that the puerperal risk of thromboembolism is highest in the immediate postpartum period. ${ }^{5}$

Furthermore, preeclampsia and caesarean section only have an impact on thrombosis risk during the puerperal

${ }^{1}$ Syed Dawood Md.Taimur, M Maksumul Haq, Saidur Rahman Khan,CM Shaheen Kabir, Hasanur Rahman, Md. Rezaul Karim, Hemanta I Gomes, Md. Salahuddin' Department of Cardiology, Ibrahim Cardiac Hospital \& Research Institute, Dhaka

Address of Correspondence: Dr. Syed Dawood Md.Taimur, Department of Cardiology, Ibrahim Cardiac Hospital \& Research Institute. Email: sdmtaimur@gmail.com

Received: 22 September, 2012

Accepted: 09 July, 2013 severe dyspnoea, haemoptysis with right leg swelling and H/O lower uteraine caesarean section(LUCS) followed by mymectomy one and half months back, initially her ECG, chest -X-ray \& echocardiographic findings revealed evidence of pulmonary embolism we treated her with dramatic improvement.

Keywords: Pulmonary Embolism ; Deep vein thrombosis (DVT); Caesarean section.

(Birdem Med J 2013; 3(2): 116-120)

period. ${ }^{3-9}$ Therefore, a higher risk level is present over a shorter time span with the risk decreasing in time. Based on this knowledge, the Swedish medical establishment has implemented a risk score model for estimating the likelihood of pregnancy-related VTE. ${ }^{10}$

The model recommends to whom, when, at what dose, and for how long thrombosis prophylaxis is recommended for pregnant women. Guidelines on the use of thromboprophylaxis have reduced deaths after cesarean delivery. ${ }^{3}$

\section{Case Report:}

A 29 year of young lady presented with progressive shortness of breath for 10 days with several episode of haemoptysis for 5 days. She has also complaints of right leg swelling and pain for 20days. She had H/O LUSC followed by mymectomy due to fibroid uterus one month back. On admission, her vital signs revealed a tachycardia of $120 / \mathrm{min}$, blood pressure of $110 / 50 \mathrm{mmHg}$ and a respiratory rate of $40 / \mathrm{min}$. Her JVP was raised swollen right leg which was tender. Precordial exam revealed that apex at normal position with left parasternal heave, normal S1, loud P2, and a pan systolic murmur of $3 / 6$ at the left lower sternal border. There was diminished breath sound in right lower lung field. Neurological exam was unremarkable.

Admission laboratory values revealed the following: hemoglobin $10.7 \mathrm{gm} \%$, white blood count $14 \mathrm{~K} / \mathrm{cumm}$, platelets $319 \mathrm{~K} / \mathrm{cumm}$, normal electrolytes, creatinine, glucose, PT and APTT. Arterial blood gases at room air 
showed $\mathrm{pH} 7.35, \mathrm{pCO}_{2} 27, \mathrm{pO}_{2} 71$, and saturation of $90 \%$. The ECG showed a sinus tachycardia with $\mathrm{S}_{1} \mathrm{Q}_{3} \mathrm{~T}_{3}$ (Fig.-1).

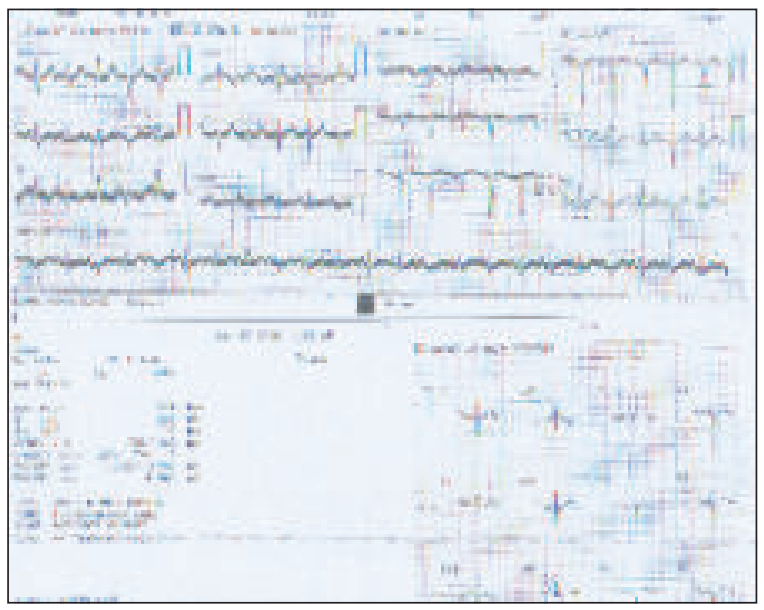

Fig.-1: ECG showed sinus tachycardia with $S_{1} Q_{3} T_{3}$.

Chest-X-ray showed wedge shaped appearance in right lateral side of lung field.Radiological diagnosis was pulmonary embolism (Fig.-2).

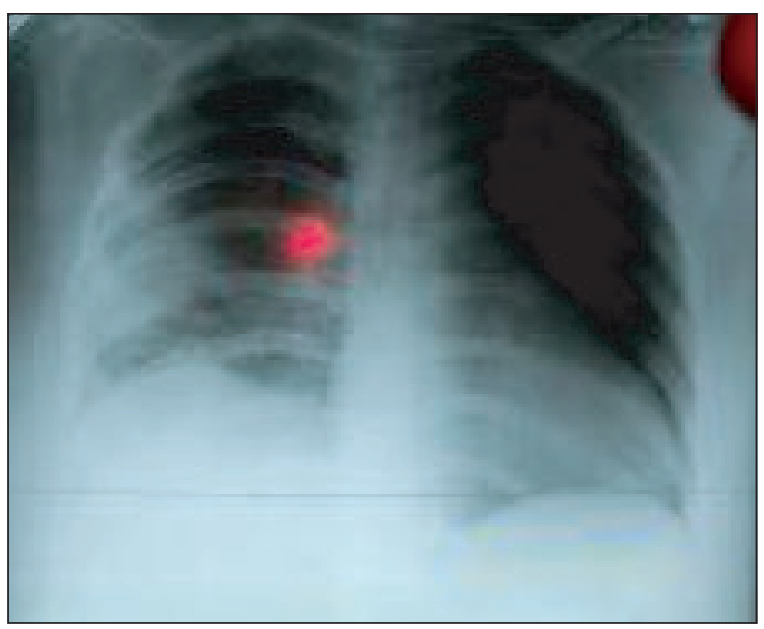

Fig.-2: Chest -X-ray showed wedge shaped opacity with pleural base in in right lower chest

Transthoracic echocardiogram showed dilatation of the right atrium, right ventricle (RVID- $3.5 \mathrm{~cm}$ ) \& pulmonary artery with moderate tricuspid regurgitation with severe pulmonary hypertension (PASP- $87 \mathrm{~mm}$ Hg)(Fig. 3a,3b)

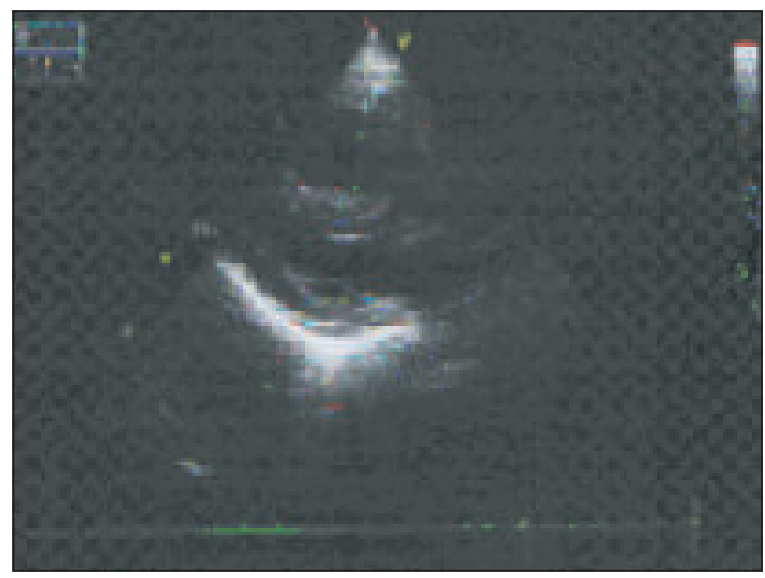

Fig.3(a)-2D-ECHO-Dilated RA

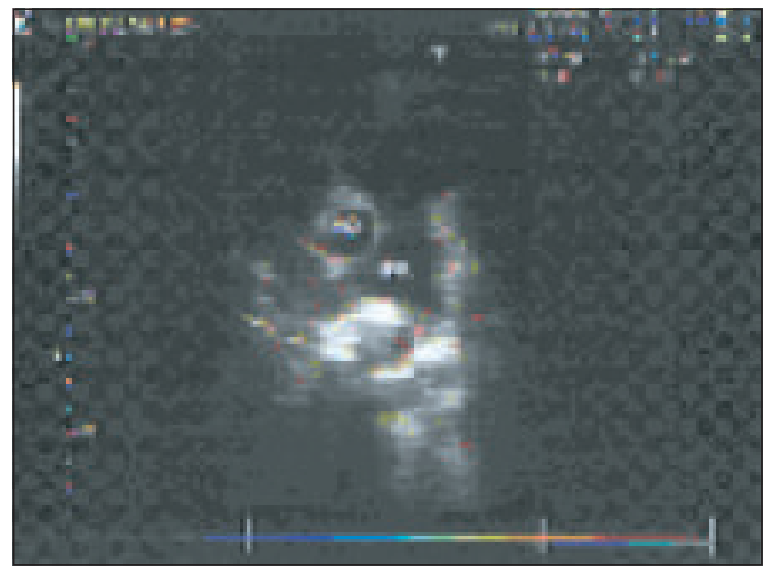

Fig.-3(b):-2D-ECHO-Dilated PA

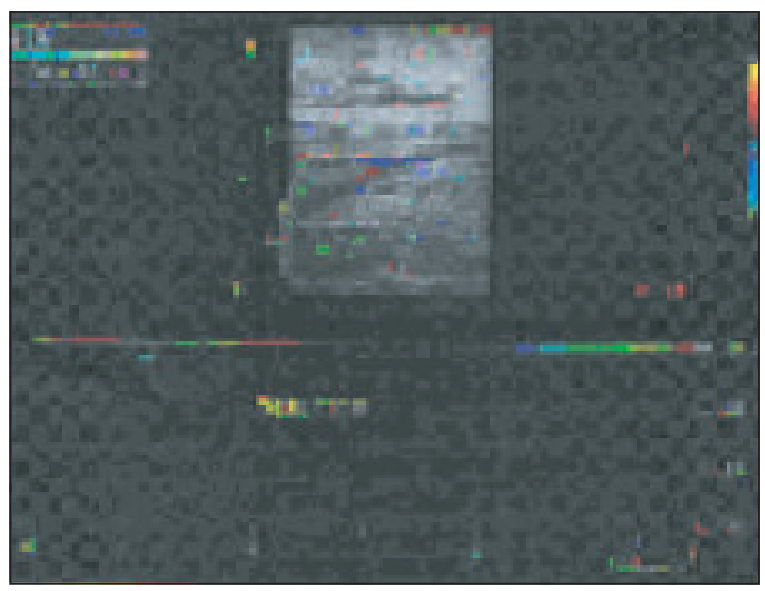

Fig.-4(a): Dupplex study of right Popliteal vein occlution. 


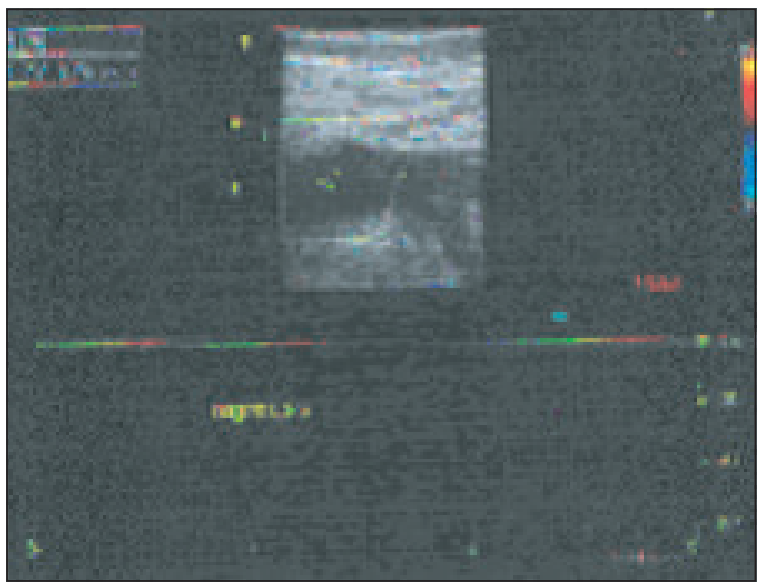

Fig.4(b): Dupplex study showed common femoral vein occlution .

Duplex study of lower limb vessels revealed(Fig.4a, 4b) : Deep vein thrombosis involving iliofemoro- popleteal (rt.) segment with out sign of recanalization. CT pulmonary angiogram revealed thrombus present in right pulmonary artery (Fig.5).

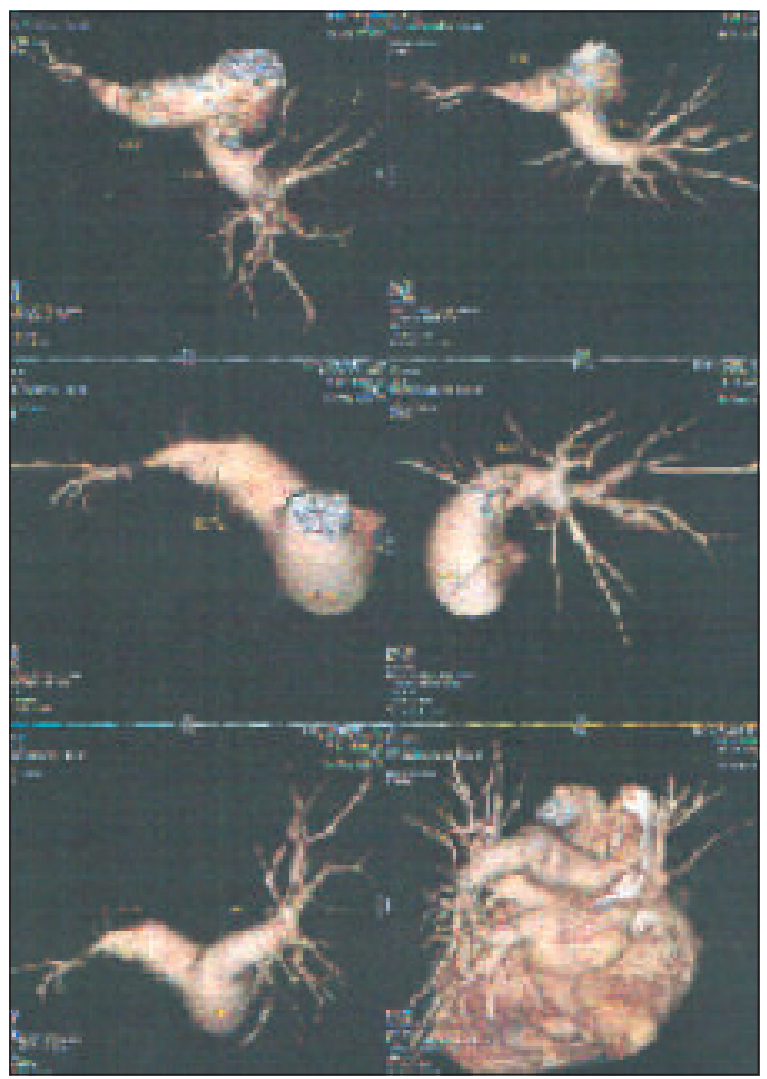

Fig.-5: CT-Pulmonary Angiogram ; Right pulmonary artery thrombus
Considering her clinical \& haemodynamic condition, thrombolytic therapy (streptokinase) was not given. She was treated with UFH 5000U/L bolus with continuous $1200 \mathrm{U} / \mathrm{hr}$ through infusion for five days and gradual improvement in her general condition. A repeated transthoracic echocardiogram showed improvement of her pulmonary hypertension as well as significant regression of the right ventricular dilatation. She was discharged with oral anticoagulant (warfarin) $5 \mathrm{mg}$ /day for 6 month with target INR should be 2.0-3.0 and was advised for repeated follow-up. After 1 month of discharge her duplex study of lower limb was with in normal limit .

\section{Discussion:}

There are three FDA approved regimens for thrombolysis in pulmonary embolism. The one preferred is the tPA regimen given as a fixed dose of $100 \mathrm{mg}$ as a continuous peripheral intravenous infusion over two hours. It is no longer believed that low to medium doses of streptokinase or urokinase are as rapidly effective or as safe. ${ }^{16}$ Bleeding is a possible side effect.

The convenience, rapid effect and relative safety of this therapeutic approach since its approval by the FDA in 1990, had led to its increasing use in the management of pulmonary embolus. Certain particular points differentiate thrombolysis for pulmonary embolism from thrombolysis for acute myocardial infarction.

According to the Thrombosis Interest Group of Canada, thrombolytic therapy may be considered for patients with massive venous thrombo-embolism (i.e. ilio-femoral venous thrombosis or pulmonary emboli with hemodynamic instability). The best results are obtained if treatment is given within the first few days from onset of symptoms. Pregnancy and head trauma or injury within the last month are relative but not absolute contraindications to thrombolytic therapy. ${ }^{3,8,12}$ Women developing VTE (venous thrombo-embolism) during pregnancy require special attention and if hemodynamically stable, heparin is usually initiated intravenously with a bolus of $5000 \mathrm{u}$ followed by a maintenance dose of 30,000 to $36,000 \mathrm{u} / 24$ hours as a continuous iv infusion, with a therapeutic range of APTT. Low molecular weight heparin may be considered as an alternative because of the lower potential for bleeding,thrombocytopenia and osteoporosis. ${ }^{6-11} \mathrm{We}$ treated our patient with UFH with oral anticoagulant and subsequently she was improved. 
Despite heightened awareness, pulmonary embolism remains a major cause of maternal mortality in the antenatal period and has not decreased in incidence over the last 30 years. Case reports about thrombolytic therapy in pregnant women are infrequent; they mainly involve steptokinase in mechanical prosthetic valve thrombosis or pulmonary emboli. ${ }^{16-23}$

There is still a risk of hemorrhage in pregnancy and particularly during delivery and post-partum, by using t-PA for thrombolytic therapy. However, it may be harmless for the fetus ${ }^{22}$ and its use appears to be safe according to the few reported cases.

Use of thrombolytic therapy should be considered when there is a great danger to the patient, however, the advantages and disadvantages should be weighed. ${ }^{22}$ It can be life saving in massive pulmonary embolism, with cardiogenic shock or overt hemodynamic instability. ${ }^{8}$ As stated, there appears to be a 14 day window for its effective administration. ${ }^{9}$ Controversy persists regarding the use of thrombolytic therapy in patients with stable systemic arterial pressure and right ventricle dysfunction. In this population, rapid improvement of RV function and pulmonary perfusion accomplished with thrombolysis, in addition to IV Heparin may lead to a lower rate of recurrence than with IV Heparin alone. ${ }^{11}$

The MAPPET Registry ${ }^{12}$ suggested that patients who are initially treated with thrombolysis plus anticoagulation had a better clinical outcome than those who were initially treated with anticoagulation alone. Despite reports and opinions regarding the benefit of surgical treatment versus thrombolytic therapy or percutaneous transvenous embolus fragmentation for massive pulmonary emboli, ${ }^{15}$ no studies exist comparing these alternatives. If aggressive intervention is warranted in patients with contra-indication to or failure of thrombolytic therapy, transvenous catheter embolectomy ${ }^{13}$ or open surgical embolectomy ${ }^{13}$ should be considered. ${ }^{23}$

\section{Conclusions:}

The presentation of acute pulmonary embolism in a previously healthy young woman who has recently given birth is an uncommon clinical scenario with some potentially fatal complications. Venous thromboembolism is uncommon but fatal condition. Echocardiography is becoming a very useful noninvasive test in the management of patients with massive pulmonary embolism. Thrombolysis or i.v heparin followed by warfarin is the treatment of choice. If diagnosed early and treated appropriately outcome is good.

\section{References:}

1. Drife J. Thromboembolism. Br Med Bull 2003; 67:17790 .

2. Drife J. Why mothers die, 2000 to 2002 report. Confidential enquiries into maternal deaths [online]. Accessed on June 10, 2008. URL: http:// www.cemach.org.uk/Publications/ Saving-Mothers-LivesReport-2000-2002.aspx

3. Lindqvist P, Dahlbäck B, Marsál K. Thrombotic risk during pregnancy: a population study. Obstet Gynecol 1999, 94:595-99.

4. Lindqvist PG, Svensson PJ, Marsál K, et al. Activated protein C resistance (FV:Q506) and pregnancy. Thromb Haemost a 1999; 81: 532-37.

5. Salonen Ros H, Lichtenstein P, Bellocco R, et al. Increased risks of circulatory diseases in late pregnancy and puerperium. Epidemiology2001; 12: 456-60.

6. Lindqvist PG, Dahlbäck B. Bleeding complications associated with low molecular weight heparin prophylaxis during pregnancy. Thromb Haemost 2000; 84: 140-41.

7. Lindqvist PG, Hellgren M, Nord E, et al.. Thrombosis prophylaxis decisions in pregnancy - simpler with risk scores. Läkartidningen 2006; 103: 1429-32.

8. Lindqvist PG, Kublikas M, Dahlbäck B. Individual risk assessment of thrombosis in pregnancy. Acta Obstet Gynecol Scand 2002; 81: 412-16.

9. Lindqvist PG, Olofsson P, Dahlbäck B. Use of selective factor V Leiden screening in pregnancy to identify candidates for anticoagulants. Obstet Gynecol 2002; 100: 332-36.

10. Lindqvist PG et al. Postpartum thromboembolism: Severe events might be preventable using a new risk score model. Vasc Health Risk Manag 2008; 4(5): 1081-87.

11. Goldhaber SZ, Haire WD, Feldstein ML, et al. Alteplase versus heparin in acute pulmonary embolism: randomized trial assessing right ventricular function and pulmonary perfusion Lancet 1993; 341:507-11.

12. Konstantinides S, Geibel A, Olschewski M, et al. Association between thrombolytic treatment and the prognosis of hemodynamically stable patients with major pulmonary embolism: results of a multicenter registry. Circulation 1997; 96: 882-88.

13. Greenfield LJ, Proctor MC, Williams DM, Wakefield TW. Long term experience with transvenous catheter pulmonary embolectomy: J Vasc Surg 1992; 18: 450-57. 
14. Gulba DC, Schmid C, Borst HG, Lichtlen P, Dietz R, Luft FC. Medical compared with surgical treatment for massive pulmonary embolism. Lancet 1994; 343:576-77.

15. Andreas Baumbach,M.D., Christiane M. Erley, M.D. Images in clinical medicine - NEJM, July 1998; 339: 86.

16. Samuel Z. Goldhaber, MD., .Recent advances in the diagnosis and lytic therapy of Pulmonary embolism. Chest 1991; 94: 173-79.

17. Tissot H. Vergnes C. Rougier P. Bricaud H Dallay D. Journal de Gynecologie Obstetrique et Biologie de la Reproduction: 1991; 20(8): 1093-96.

18. Bulent Goreneck, MD et al . Case of biatrial thrombosis. Echocardiography: Am. Jrnl of CV Ultrasound \& Allied Tech.1998; 15(6): 5-7.
19. Ginsberg and Hirsh. Chest 1995; 108 (Suppl): 305 .

20. Ramamurthy S. American Heart Journal.1994; 127(2): 446-48.

21. Samuel Z. Goldhaber MD, William R. Auger ,MD : Treatment of massive pulmonary embolism .ACCCurrent Journal Review 1998;7(5): 49-51.

22. Samuel Z Goldhaber, Luigi Visisan, Marisa DeRosa. : I.C.O.P.E.REGISTRY. Lancet 1999; 353: 9162.

23. Chan L,MD. Cutler A. MD.Thrombolytic therapy and prophylactic anticoagulation in pregnant patients. The American Journal of Emergency Medicine 1999; 17(1):107-108. 